\title{
A DEEP SEARCH FOR THE HOST GALAXIES OF GRBS WITH NO DETECTED OPTICAL AFTERGLOW
}

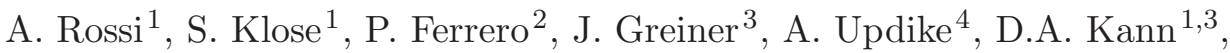 \\ T. Krühler ${ }^{5}$ and A. Nicuesa Guelbenzu ${ }^{1}$
}

\begin{abstract}
Long gamma-ray bursts (GRBs) pinpoint star-forming galaxies as they are linked to the deaths of massive stars. In most cases, these galaxies have been found to be blue, sub-luminous and of lowmetallicity. However, our recent survey have demonstrated that a sizeable fraction of GRBs reside in massive, dusty and star-forming extremely red objects (EROs, $\left.(R-K)_{\mathrm{AB}}>3.5\right)$. The most remarkable case is the host of GRB 080207, one of the reddest galaxies ever associated with a GRB. This discovery suggests that at least a fraction of GRB afterglows trace a subpopulation of massive starburst galaxies, which are markedly different from the most studied blue, sub-luminous and compact GRB host galaxies.
\end{abstract}

\section{Introduction}

Gamma ray bursts (GRBs) are the most luminous phenomena in the Universe, coming from remote galaxies up to redshift $z \sim 8$ (e.g., Salvaterra et al. 2009; Tanvir et al. 2009). Among all the detected GRBs, about 90\% are long GRBs lasting more than two seconds and signal the death of a very massive star. GRB hosts $(\mathrm{GRBHs})$ are better known in the low-z regime (up to $z \lesssim 1.5$ ), where they have been found to be sub-luminous, blue, with low metallicity and moderate star formation (e.g., Savaglio et al. 2009). However, recent surveys showed that this is just a fraction of the host population (Hjorth et al. 2012; Rossi et al. 2012).

Up to $30 \%$ of long bursts are classified as dark GRBs. A GRB is considered as a dark burst if the optical flux is lower than expected when extrapolating the X-ray flux to the optical bands (e.g., Jakobsson et al. 2004). In Greiner et al. (2011) we

\footnotetext{
1 Thüringer Landessternwarte Tautenburg, Sternwarte 5, 07778 Tautenburg, Germany

2 Instituto de Astrofísica de Canarias (IAC), 38200 La Laguna, Tenerife, Spain

3 Max-Planck-Institut für Extraterrestrische Physik, Giessenbachstraße, 85748 Garching, Germany

${ }^{4}$ Department of Physics and Astronomy, Dickinson College, Carlisle, PA 17013, USA

5 Dark Cosmology Centre, Niels Bohr Institute, Univ. of Copenhagen, Juliane Maries Vej 30, 2100 Købnhaven, Denmark
} 

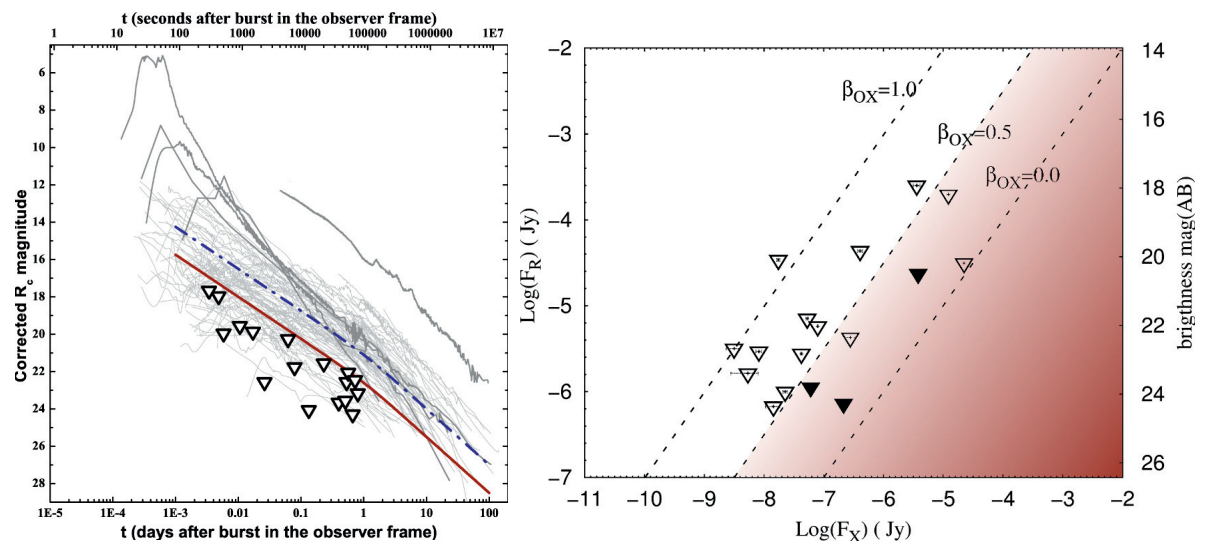

Fig. 1. Left: the $R_{C}$-band light curves of all (long) afterglows in the sample of Kann et al. $(2010,2011)$. All data have been corrected for Galactic extinction. Triangles indicate equivalent $R_{C}$-band upper limits of the afterglows in our sample. The blue dashed/dotted line approximately indicates the mean of the afterglow brightness distribution. The brown solid line indicates the border line of all targets. Right: observed upper limits in the $R_{c}$ band compared to the measured flux density at $1.73 \mathrm{keV}$ (the logarithmic mean of the Swift/XRT window, $0.310 \mathrm{keV}$ ) for the 17 bursts in our sample. The bursts falling in the gray area are dark according to Jakobsson et al. (2004).

show that extinction by dust in combination with a modest redshift is the main cause of the optical dimness of dark GRBs. However, their host galaxies, especially those of the most extinguished ones, are poorly studied, because observations are mainly limited to the optical bands.

Here, we focus on the search for the host galaxies of a sample of 17 long GRBs with arcsec-sized Swift/XRT error circles but no detected optical/NIR afterglow in spite of deep and rapid follow-up observations. Our study is based on deep $R_{C}$ and $K_{s}$-band observations performed at the ESO/VLT, partly supported by observations with the seven-channel imager GROND (Greiner et al. 2008) at the 2.2-m telescope on La Silla and other facilities. Firstly, we verify whether the bursts can be classified as dark GRBs. Afterwards, we identify the host-galaxy candidates and study their color properties to check if these can be different from the hosts of optically bright GRBs.

\section{Results}

Six events, namely GRBs 050717, 050922B, 070429A, 080207, 080218B, and 080602, are dark according to Jakobsson et al. (2004) (Fig. 1, right).

For 15 of the 17 bursts we find at least one galaxy inside the doubled XRT error circle, in two cases only a deep upper limit in $R_{C}$ and $K_{s}$ can be provided. In seven cases we discover extremely red objects (EROs; Elston et al. 1988) in the error circles, at least four of them might be dust-enshrouded galaxies. The 


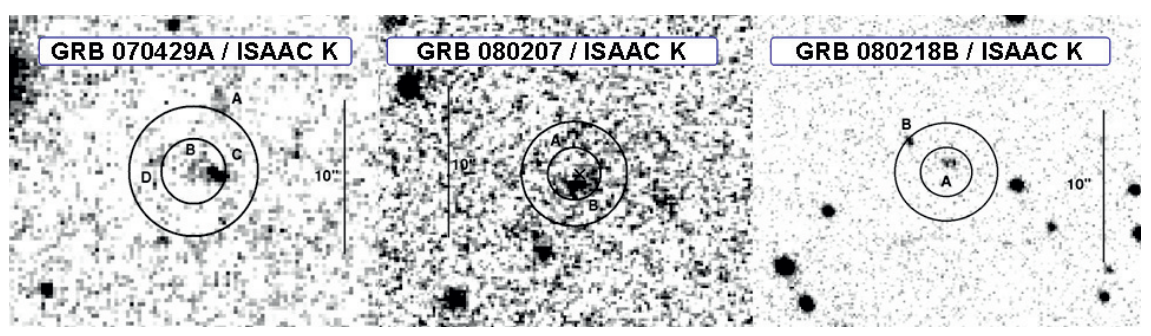

Fig. 2. The figures show the VLT/ISAAC $K$-band images of the ERO host galaxies of three dark GRBs in the sample.

most remarkable case is the host of the dark GRB 080207 which has a colour of $\left(R_{C}-K_{s}\right)_{\mathrm{AB}} \sim 4.7 \mathrm{mag}$, therefore it is the reddest galaxy ever associated with a GRB. Thanks to our results we doubled the known number of EROs hosting GRBs. On average these galaxies differ from the blue, sub-luminous, low-metallicity host galaxies that are normally associated with optically bright GRBs (e.g., Savaglio et al. 2009). Instead, their color recall those of ultra-luminous infrared galaxies or even sub-millimeter galaxies (e.g., Pope et al. 2005).

\section{Conclusions}

Optically dim afterglows result from cosmological Lyman drop out and dust extinction, but the former process is only required for a minority of cases $(\lesssim 1 / 3)$. Extinction by dust in the host galaxies might explain all other events. Thereby, a seemingly non-negligible fraction of these hosts are globally dust-enshrouded, extremely red galaxies. This suggests that bursts with optically dim afterglows trace a subpopulation of massive star-burst galaxies, which are markedly different from the main body of the GRB host galaxy population, namely the blue, sub-luminous, compact galaxies.

\section{References}

Elston, R., Rieke, G.H., \& Rieke, M.J., 1988, ApJ, 331, L77

Hjorth, J., Malesani, D., Jakobsson, P., et al., 2012, ApJ, 756, 187

Greiner, J., Krühler, T., Klose, S., et al., 2011, A\&A, 526, A30

Greiner, J., Bornemann, W., Clemens, C., et al., 2008, PASP, 120, 405

Jakobsson, P., Hjorth, J., Fynbo, J.P.U., et al., 2004, ApJ, 617, L21

Kann, D.A., Klose, S., Zhang, B., et al., 2010, ApJ, 720, 1513

Kann, D.A., Klose, S., Zhang, B., et al., 2011, ApJ, 734, 96

Pope, A., Borys, C., Scott, D., et al., 2005, MNRAS, 358, 149

Rossi, A., Klose, S., Ferrero, P., et al., 2012, A\&A, 545, A77

Salvaterra, R., Della Valle, M., Campana, S., et al., 2009, Nature, 461, 1258

Savaglio, S., Glazebrook, K., \& Le Borgne, D., 2009, ApJ, 691, 182

Tanvir, N.R., Fox, D.B., Levan, A.J., et al., 2009, Nature, 461, 1254 
